\title{
INVESTIGATIONAL INSIGHT ON GAUSSIAN AND GAUSSIAN-HALO DOPED DOUBLE GATE MOSFETS
}

\author{
R P Ramesh ${ }^{1}$, G C Vivek Chakaravathi ${ }^{1}$ \\ ${ }^{1}$ UG Final Year Students, Department of Electronics and Communication Engineering
}

\begin{abstract}
The performance based analysis for typical Gaussian and Gaussian-Halo Doped Double Gate MOSFETs in conjunction with Normal Doped DG MOSFETs, for which different parameters such as Oxide Thickness, Ambient Temperature, Gate Material Work Function and Substrate Doping Concentration is varied. This analysis has been carried out using a TCAD Lab simulation.. From the results obtained, it will be quite clear that the sub-threshold leakage current of the Gaussian and Gaussian-Halo Doped Double Gate Metal Oxide Semiconductor FET is relatively lesser. Furthermore the results have been plotted with different Drain voltage values to enhance the understanding of the physics behind various Doping Profiles in Double Gate MOSFET. Finally the results has been obtained, analysed and compared. The Possession of higher Drain current Performance is very acceptable through the rigorous analysis using a TCAD Lab.
\end{abstract}

\section{KEYWORDS}

Gaussian Doping, Halo Doping, Double Gate MOSFET, Tunnelling, Reverse Saturation current, Work Function, Drain current performance.

\section{INTRODUCTION}

In the past 40 years with downscaling, silicon transistors have become smaller and smaller in accordance to Moore's law (1965), which predicted a decrease in feature sizes by a factor of 0.7 every three years. Dr. Gordon E. Moore the entrepreneur and co-owner of the INTEL Corporation had predicted the fate of semiconductor size evolution in 1965. It states that 'the number of Transistors in a sole semiconductor chip Doubles every two years' i.e. there will be an exponential decay in the rate of size of semiconductors. While this law standing still today, the prime purpose of every world class design Engineer in the making of a real time application system using sizable section of electronics is to inject the right component at the right area [1]. This is taken as the literary oath in the initial stage of the research by the authors that helped in bringing out the tip to top analysis of the widely used peculiar variety of Gaussian and GaussianHalo Doped DG MOSFETs to draw the drawbacks and merits on operation of the indicated transistor in different internal and external environment around the MOSFET..

In Hi-Fi application processing equipment, the accuracy of the transistor operation is of utmost concern in its final mass performance. The sub-threshold leakage on such scenario has to be shrunk in serving the semiconductor industry in a sensible way thereby supporting healthy system. Other Doping Levels of the MOSFETs such as uniformly doped, Un-doped and Lightly Doped MOSFETs has its own drawbacks in the form of inaccuracy during its modelling[1].This is mainly because the modelling of perfectly flat doping channel is bizarre due to the presence of Residual Impurities in the MOSFET which typically gives rise to its unique Flat Band Voltage

DOI : $10.5121 /$ ijist.2014.4310 
[1]. Moreover there is no barricade between source and drain of the un-doped channel MOSFET which makes it prone to exhibit noisy response [2]. Contradictorily in case of Gaussian and Gaussian-Halo Doping, the Drain current performance can be enhanced and it has lower sub threshold leakage over the other doping of the MOSFETs. The important criteria of any engineering project is that the accuracy of the component being used. Gaussian and GaussianHalo Doped transistor made of Metal Oxide semiconductor ensures the sensation on that which is being confirmed by the successive exhibitions of simulations.

Threshold voltage of the Gaussian Doped DG MOSFET is twice of its Fermi Potential Profile. This possesses different threshold voltages in this Gaussian Doping Profile and it is quite obvious that the surface with lower potential turns ON first over the surface with higher potential $[3,4]$. This is due to its work function variation between the Gate and Channel in its various sections. This will have the direct effect on the Threshold voltage of the DG MOSFET at which Inversion occurs. Moreover it contributes to the sub-threshold leakage current. All these analysis has been carried out thoroughly in the successive parts of the proposed work by the authors.

\section{MODEL INCARNATION AND PROPOSED WORK}

The schematic view of the contour plot of the Double gate metal oxide semiconductor field effect transistor is shown in the figure 1(a).Thedepiction of figure 1(a) shows the Gaussian doping level on the Double Gate MOSFET. The schematic view of the 2D-Double Gate MOSFET is shown below in the figure 1(b). In our paper, we have investigated the width and breadth of the performance of Gaussian and Gaussian-Halo Doped Double Gate MOSFETs [5].
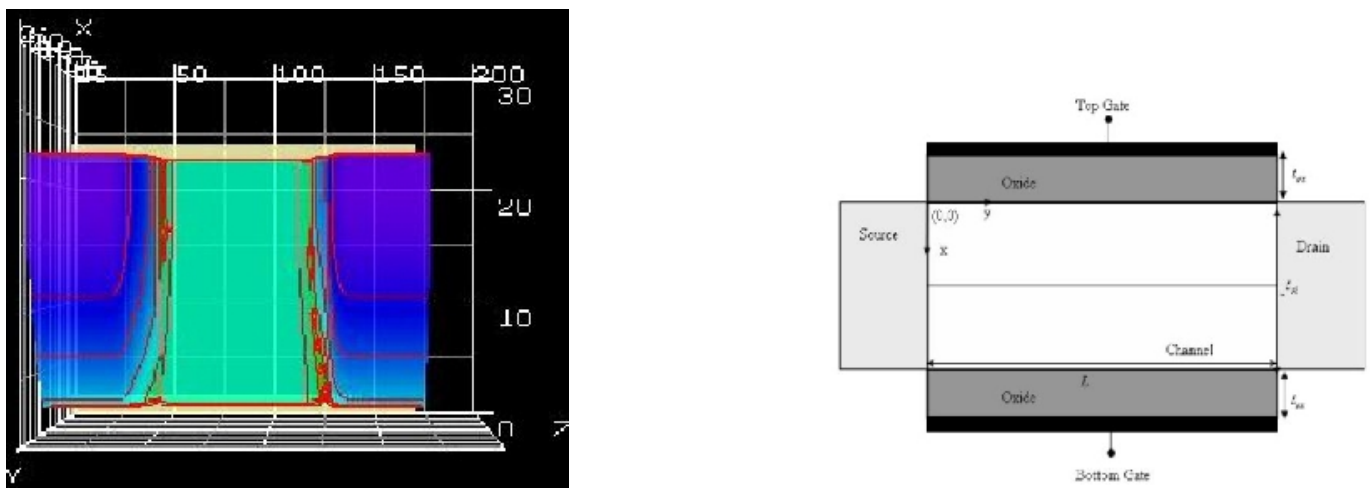

Figure 1(a) Contour 3D-Gaussian DG MOSFETFigure 1(b) Schematic view - DG-MOS

Moore's Law has been satisfied by the semiconductor industry since 1965. In the scaling era of MOSFET, the main drawback in its performance arises due to its intended short channel effects in the form of Drain Induced Barrier Lowering (DIBL), Hot Carrier Effects, Velocity Saturation and Impact Ionization effects [7, 8]. These SCEs lead to severe degradation in the performance of the transistor. If the Drain to source voltage increases beyond a particular voltage known as Pinch-off voltage, then the channel near the drain region tends to pinch off. This is due to the heavy Reverse bias across the Drain and Substrate which tends to increase the Depletion across them to extend into the inversion region [9]. This seriously affects the performance of the transistor since the contact potential across the depletion capacitance enhances the electron swing around the drain region with Reverse saturation leakage current. This indeed leads to the Non-Linear characteristics between Drain current and Gate Voltage which is highly undesirable. One of such effects is DIBL i.e. the Drain voltage taking over the Gate voltage in controlling the Drain 
current. As the electric field across the depletion region increases during the higher drain voltage, this moreover leads to reduction of the mobility of electron and velocity saturation [1]. This furthermore hot electrons leads to strike lattice atoms and ionize them i.e. breaking the valence electron of the lattice atoms and it is being an avalanche process. Generally, the scattering inside the metal oxide semiconductor FET is due to impurity scattering and lattice scattering.

In order to control these undesirable effects, an extra Gate can be fabricated on the back side of the MOSFET [1]. This leads to Double Gate MOSFET. This ensures better control over channel which can relatively minimise the short channel effects. As this have several advantages such as Low Noise, Better Performance and Preservation of Linearity over Id-Vgs characteristics over larger range, this type of amplifier steals the attention of modern world engineers. This solid performance of the DG-MOS combined with Gaussian and Gaussian-Halo Doping Profile gives furthermore opportunities to scrutinize the control systems in the real world applications.

Moreover Gaussian and Gaussian-Halo Profile Guarantees lesser Sub-Threshold current which is otherwise known as OFF-current. Mathematical Gaussian Doping Profile mathematical model [2] can be given by equation (2).

$$
\left.N_{b}(x)=N_{p} c(a+2 b \propto X)^{2}-2 b\right] e^{\left(-a \alpha X-b X^{2}\right)}
$$

Where $\mathrm{X}=\frac{x-R_{p}}{x_{p}}$;

$x_{b}=\sqrt{2} \sigma_{p}$

$R_{p}$ Being the Projected Range

$\sigma_{\mathrm{p}}$ Being the straggle of the Gaussian Profile

$\mathrm{N}_{\mathrm{p}}$ Being the peak doping concentration at $\mathrm{x}=R_{p}$

$\mathrm{a}, \mathrm{b}, \mathrm{c}$ are the fitting constants

Here, the Doping Profile linearly varies from source to drain approximating the mathematical model of Gaussian distribution with mean $\mu$ and standard deviation $\sigma$. In this paper, the authors emphasized their interest on Gaussian and Gaussian-Halo Doping Profile analysis with different parameters and deducted I-V characteristics analysis. It has been deducted for two different drain to source voltage to enhance the chances of understanding the nature of its behaviour. Moreover the survey conducted on other doping profile reveals straight away that the sub-threshold leakage on Gaussian Doped DG MOSFET is far lesser. This analysis is elongated with simulations and comparison for Normal and Gaussian-Halo Doped DG MOSFET under varying Oxide Thickness, Ambient Temperature, Gate Material Work Function and Substrate Doping Concentration.

In order to reduce the undesirable SCE (Short Channel Effects), Halo Doping is usually employed. Doping of high amount of impurities in a shallow manner under source and drain is known as Halo Doping. When the MOS-Transistor is not halo-doped, the depletion region across the PN-Junctions of the MOSFETs will exhibit some reverse saturation current which mostly depends on the ambience temperature. Due to the heavy doping, PN-Junction Depletion Region decreases which also decreases the reverse saturation current influenced due to them [1]. Combination of Gaussian and Halo Doping gives rise to very less leakage current. Hence the expected result is the reduction in the overall leakage current. 


\section{RESULTS AND DISCUSSIONS}

In an endeavour to analyse the effectiveness of Gaussian-Halo Doped Double Gate MOSFET in the making, the Gaussian Doped DGMOS, Gaussian-Halo Doped DG MOS and Uniformly Doped normal DG MOS has been studied, compared and the results has been depicted. Moreover the results are compared and deduced using TCAD Lab.

Hence, here by the authors decided to analyse the Gaussian and Gaussian-Halo Doped DG MOSFETs Drain current characteristics and its variations due to several parameters. The result has been compared with the identical normal doping (Uniformly Doped) MOSFET. The Gate material is indeed important in controlling the inversion regime of the MOSFET. It models the threshold voltage of its particular which is very likely on the difference of work functions between the gate and channel potential. So the metals such as Aluminium, Tungsten, Silver (Poly Crystalline) has been used as Gate Material and the resultant I-V characteristics readily emphasizes the advantages of different parameters on different circumstances. Certainly another important criteria for an industrial purpose MOSFET is that its response under different temperature conditions.

Mainly, the MOSFET usage area can be classified under three categories via piecewise applications, (i) Industrial purpose (ii) Military purpose. Inevitably the military purpose applications should have the equipment with high withstanding capability against noise under different environment temperature conditions in order to sustain the accuracy. This is of utmost importance which will satisfy the primary concern of the industry. Hence the brief survey has been carried out by the authors especially on these two factors.

For the purpose of simplicity, the radiation effects and scattering effects inside the MOSFET has been neglected. Furthermore, the complete analysis of Gaussian and Gaussian-Halo Doping Profile based DG MOSFET over Normal Doped DG MOS has been scrutinized and compared.

The Graphs obtained for Different Oxide Thickness, Ambient Temperature, Gate Electrode Work Function and Substrate Doping Concentration for Normal, Gaussian and Gaussian-Halo Doped Double Gate MOSFETs is being followed.

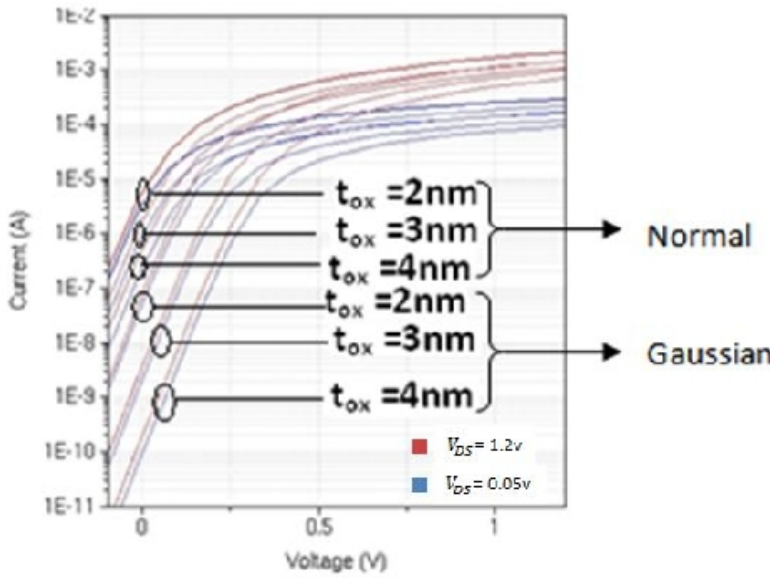

Figure 2(a). I-V-Characteristics-Gaussian-Doped Doped DG MOS-Oxide Thickness

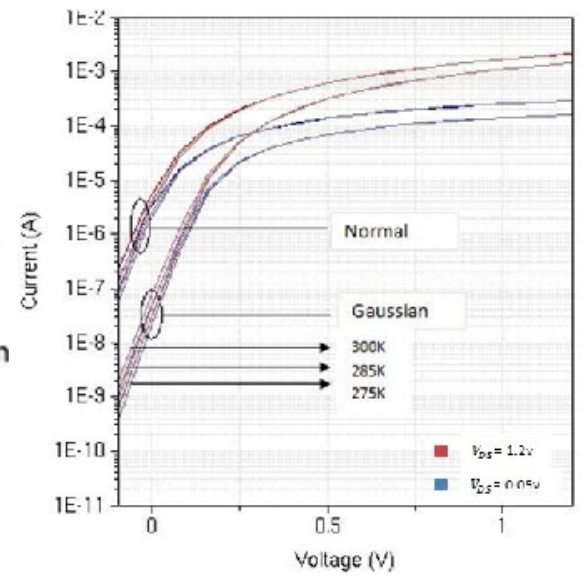

Figure 2(b). I-V-Characteristics- Gaussian Doped DG MOS-Ambient Temperature 
Figure 2(a). shows the dependence of Drain current and inversion channel regime variation due to the dielectric oxide thickness. For better understanding of the Id-Vg Characteristics of MOSFET with respect to different oxide thickness has been simulated thoroughly with different drain voltage values. Here in the simulation, $V_{d}=0.05$ volts and $V_{d}=1.5$ volts are taken for the analysis of its performance. There is a primary theory in the making that the Drain current performance of the MOSFET gets decreased when Thickness of the dielectric oxide is increased. This intuitively increases the Gate voltage to cause the channel inversion. Here $t_{o x}$ is varied from $2 \mathrm{~nm}$ to $5 \mathrm{~nm}$ to compare the performance of Normal Doped and Gaussian Doped DG MOSFETs. From the graph plotted, it has been very clear that the leakage current of the Gaussian Doped MOSFET is relatively lesser than that of the Normal Doped MOSFET.

Figure 2(b). shows the behaviour of DG MOSFET under different ambient temperature in both Normal and Gaussian Doped version. From the graph, it will be very precise that higher the ambient temperature leads to higher the sub-threshold leakage current. This tends to turn ON the transistor before the expected threshold setting of it. However the Gaussian Doped DG MOSFET possesses lesser leakage current than that of the Normal Doped version. This ambient Temperature analysis has been carried out since the MOS-Transistor used in an IC-Chip has to be accurate for the entire chip element to be salubrious. Also the Equipment such as Military PC's will be used for security purpose even under zero Degrees Centigrade or above 30 degrees centigrade. At those instants, the MOS-Transistor should possess lesser leakage current in order to confirm the sustainable nature of the Device at various environments.

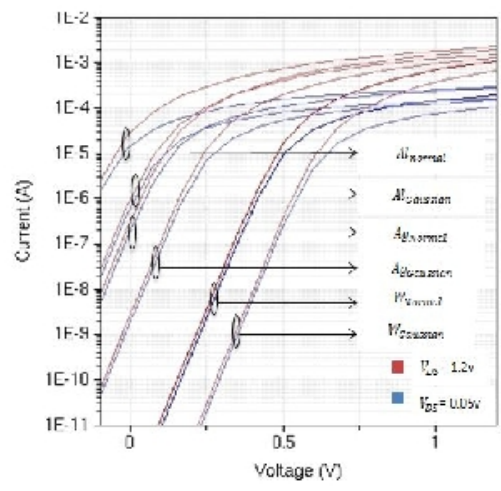

Figure 2(c). I-V-Characteristics of Gaussian Doped DG MOS with varying Gate Material.

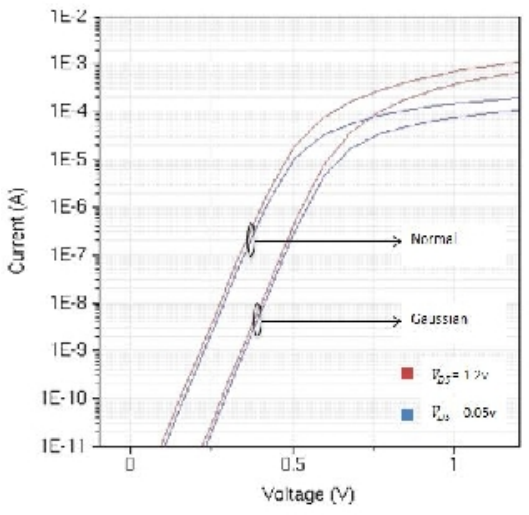

Figure2(d) I-V-Characteristics of Gaussian Doped DG MOS with varying Substrate Doping Concentration.

Figure 2(c). shows the Detailed Results for Gaussian doped DG MOSFET is obtained for materials such as Aluminium (Al), Silver (Ag) and Tungsten (W) for both Normal and Gaussian Doped DG MOSFET. The work Function of the respective metals is given by $4.08 \mathrm{eV}, 4.26 \mathrm{eV}$ and $4.55 \mathrm{eV}$. However Aluminium is used in the beginning of the MOSFETs era, the reason for Tungsten being the most widely used Gate Material is quite clear from the graph that it will provide lesser leakage current over other Materials. Moreover, this ensures that the Gaussian doping has further lesser leakage current than that of the normal doped DG MOSFET.

Figure 2(d). shows the result obtained for Drain current characteristics of both Normal and Gaussian Doped DG MOSFETs. Two different drains to source voltages has been kept in predicting the graph to provide better insight in understanding the physics behind the operation of the DG MOSFET under different doping concentrations. Due to the leakage flux induced around the environment, the noise might induce the Normal Doped MOSFET into ON-Condition without the application of Gate Voltage. This might not occur in terms of Gaussian Doped DG MOSTransistor since the knee voltage is a bit lag from the Normal Doped DG MOSFET. 


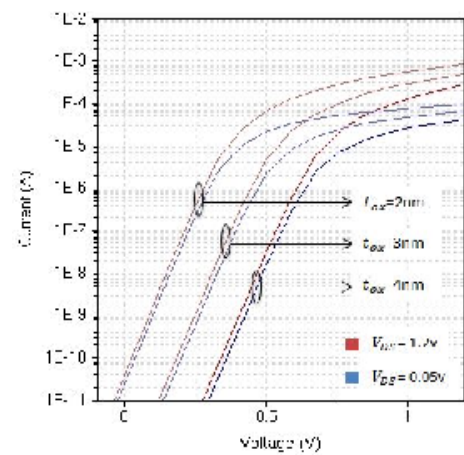

Figure 3(a). I-V-Characteristics of Gaussian Doped DG MOS with varying Oxide Thickness.

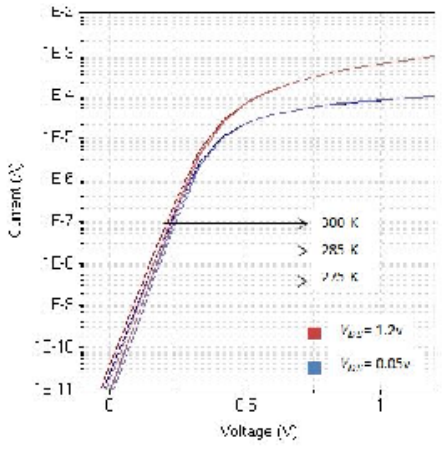

Figure3(b) I-V-Characteristics of Gaussian Doped DG MOS with varying Ambient Temperature.

Figure 3(a). shows the Graph that portrays the Drain current Performance characteristics for Gaussian come Halo Doped DG MOSFETs. Halo Doping is especially used in order to reduce SCE (Short Channel Effects). Due to this, the overall tunnelling will be far lesser than that of Gaussian Doped Double Gate MOSFETs. Also it is quite understandable that the tunnelling leakage is lesser when the oxide thickness between the gate and channel becomes furthermore less.

Figure 3(b). shows the graph which is the Drain current characteristics of Gaussian come halo doped Double gate MOSFETs for Different Environment temperature such as $300 \mathrm{~K}$ (Room Temperature), $285 \mathrm{~K}$ and $275 \mathrm{~K}$. It is again very likely that the leakage current in Gaussian-Halo Doped Double Gate MOSFET is lower than that of the Gaussian Doped DG MOSFET.

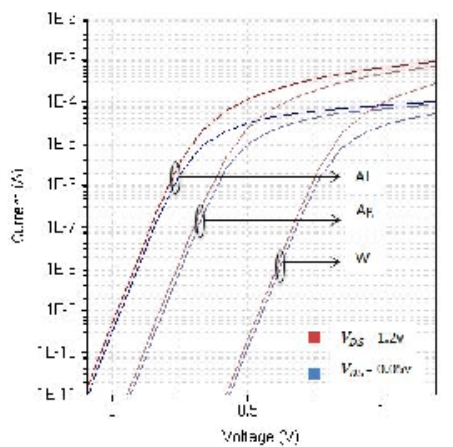

Figure 3(a). I-V-Characteristics of Gaussian Doped DG MOS with varying Gate Material.

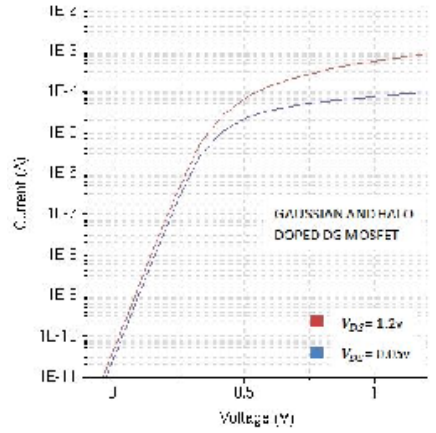

Figure3(b) I-V-Characteristics of Gaussian Doped DG MOS with Substrate Doping Concentration.

Figure 3(c). depicts the result showcases the Drain current characteristics of the Gaussian-Halo Doped Double Gate MOSFETs with Different Gate Material Work Function. Threshold voltage is defined as the voltage at which the MOSFET turns ON. It depends on the Work Function Difference between the Gate and Channel. From this result, it can be declared that Tungsten is appropriate metal for providing improved Drain current performance.

Figure 3(d).Portrays the results obtained for Drain current performance of the Gaussian come Halo doped Double Gate MOSFETs. This is compared with the Normal and Gaussian Doped Double Gate MOSFETs Graph. From the results, it will be quite clear again that the Gaussianhalo doping provides furthermore lesser leakage current and improved drain current performance. 


\section{CONCLUSION}

The proposed work legitimately deals with Normal, Gaussian and Gaussian-Halo Double Gate Metal Oxide Semiconductor Field Effect Transistor (DG-MOSFET) and its Drain current performance characteristics with different parametric variations. The parameters such as Dielectric Oxide Thickness, Ambient Temperature, Gate Material Work Function and Substrate Doping concentration has been varied to provide the better understanding of the physics behind the MOSFET in this regime. From the results obtained, it can be inferred that Tungsten can provide lesser leakage current than that of the other materials, Drain current performance under Different temperature and neat exhibition of Tunnelling effect. Finally, substrate doping concentration such as Normal, Gaussian and Gaussian-Halo Doped DG MOSFETs is indulged in the analysis. The results obtained infer that the Drain current performance is quite higher in the order of Gaussian-Halo, Gaussian and Normal Doped Double Gate MOSFET.

\section{REFERENCES}

[1] Yannis Tsividis, Operation and Modeling of MOS Transistors. United Kingdom: Oxford University Press, 2007.

[2] Sarvesh Dubey, Pramod Kumar Tiwari and S. Jit, "A Two Dimensional model for the Potential Distribution and Threshold voltage of short-channel double gate metal oxide semiconductor", Journal of Applied Physics 108, 034518 (2010).

[3] Pramod Kumar Tiwari and S. Jit, "A Sub-threshold swing model for Symmetric Double Gate (DG) MOSFETs with Vertical Gaussian Doping”, Journal of Semiconductor Technology and Science, Vol.10, NO.2, June 2010.

[4] Sarvesh Dubey, Pramod Kumar Tiwari and S. Jit, "On-current modelling of short-channel double-gate (DG) MOSFETs with a vertical Gaussian-like doping profile”, Journal of semiconductor, Vol., 34, No.5,2013.

[5] A. Nandi, A. K. Saxena, t al, "Analytical Modelling of a Double Gate MOSFET Considering Source/Drain Lateral Gaussian Doping Profile", IEEE Transaction on Electron Devices, Vol., 60, Issue 11, 2013.

[6] Petru Andrei, Isaak Mayergoyz, "Random Doping Fluctuations of Sub-Threshold characteristics in MOSFET Devices", Solid State Electronics Elsevier Journal 47 (2003).

[7] T. K. Chiang, "A new compact sub-threshold behaviour model Dual-Material Surrounding Gate (DMSG) MOSFETs”, Solid State Electronics Elsevier Journal 53 (2009).

[8] Hamdy Abd El Hamid, JaumeroigGuitart and Benjamin Infguez, "Two-Dimensional Analytical Threshold Voltage and Sub-threshold Swing Model of Un-doped Symmetric Double-Gate MOSFETs", IEEE Transaction on Electron Devices, Vol., 54, No. 6, June 2007.

[9] Pujarani Ghosh, SubhasisHaldar, R. S. Gupta, Mridula Gupta, “An Analytical Drain current model for Dual Material Engineered Cylindrical/ Surrounded Gate MOSFET", Microelectronics Journal 43 (2012).

\section{Authors}

R. P. Ramesh. He pursues B .E inElectronics and Communication Engineering in Velammal College of Engi-neering and Technology, Madurai. He is interested in nanoscale MOS devices.

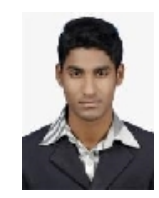

G. C. Vivek Chakaravarthi. Hepursues B .E in Electronics and Communication Engineering in Velammal College of Engineering and Technology, Madurai. He is interested in analog V LSI. 